Research Article

\title{
Exact Overlap Rate Analysis and the Combination with 4D BIM of Time-Cost Tradeoff Problem in Project Scheduling
}

\author{
Guofeng Ma and Lingzhi Zhang $(\mathbb{D}$ \\ School of Economics and Management, Tongji University, Siping Road No. 1500, Shanghai 200092, China \\ Correspondence should be addressed to Lingzhi Zhang; zlz1631031@tongji.edu.cn
}

Received 16 January 2019; Revised 16 March 2019; Accepted 2 April 2019; Published 2 May 2019

Academic Editor: Behzad Esmaeili

Copyright (c) 2019 Guofeng Ma and Lingzhi Zhang. This is an open access article distributed under the Creative Commons Attribution License, which permits unrestricted use, distribution, and reproduction in any medium, provided the original work is properly cited.

\begin{abstract}
Schedulers can compress the schedule of construction projects by overlapping design and construction activities. However, overlapping may induce increased total cost with the decrease of duration. To solve the concurrency-based time-cost tradeoff problem effectively, this paper demonstrates an overlapping optimization algorithm that identifies an optimal overlapping strategy with exact overlap rates and generates the required duration at the minimum cost. The method makes use of overlapping strategy matrix (OSM) to illustrate the dependency relationships between activities. This method then optimizes the genetic algorithm (GA) to compute an overlapping strategy with exact overlap rates by means of overlapping and crashing. This paper then proposes an integrated framework of genetic algorithm and building information modeling (BIM) to prove the practice feasibility of theoretical research. The study is valuable to practitioners because the method allows establishing a compressed schedule which meets the limited budget within the contract duration. This article is also significant to researchers because it can compute the optimal scheduling strategy with exact overlap rates, crashing degree, and resources expeditiously. The usability and validity of the optimized method are verified by a test case in this paper.
\end{abstract}

\section{Introduction}

The demand for a shorter project completion time has led to a variety of techniques adopted on schedule compression in the construction field: activity crashing, substitution, and overlapping $[1,2]$. The overlapping of activities accelerates the execution of engineering projects by executing two activities in parallel [3]. However, starting a successor without complete upstream information may introduce the risk of rework to the successor to accommodate unforeseen upstream developments [4]. Rework in turn will induce unnecessary cost and lengthen the duration. On the other hand, crashing can compress the schedule a lot with additional resources and labor, but the accompanying cost is quite high. Hence, it is certain that a tradeoff between time and cost is important to the scheduler who attempts to accelerate a project within required duration or cost.

To date, there are many studies on time-cost tradeoff in schedule compression $[5,6]$. In the research of overlapping, most of current researchers calculate the lap time based on an empirical formula. There is no tool for designing, measuring, and evaluating the overlapping strategy that has the potential to shorten the schedule precisely with exact overlapping rate in building construction projects. In the research of crashing, most studies assume that the relationship between time and cost is linear. The research under this condition is not in accordance with the reality. And, the existing theoretical research is not combined with practical projects or software, so it is difficult to prove their practical feasibility and dynamic control effect. Set against this is the fact that the society is developing and changing rapidly so that time is valued far beyond imagination and the schedule needs to be as accurate as possible and convenient to be adjusted in practical construction activities. Therefore, this kind of tool would be significant for schedule optimization and rework reduction in construction projects. Recent work has shown various time-cost tradeoff methods proposed to deal with the overlapping and crashing issues. However, very few researchers provide an appropriate 
method to calculate the rework duration and cost, few identify a mathematical model that quantifies the overlap rate accurately, and hardly any researcher associate it with crashing and combine traditional algorithm with BIM platform to realize second optimization and dynamic control.

Here, this paper describes the mechanism of a combinatory model in which the construction information can be precisely delivered into and whereupon subsequently an optimal scheduling strategy of this construction project can be obtained. Distinct from the previous reports, the presented model can give out a precise overlapping rate about what percentage of an activity should be overlapped for the optimal solution and the accurate crashing rate with a completely new method of integrating the genetic algorithm with Overlapping Strategy Matrix. Apart from the first optimized overlapping strategy, the authors proposed a framework combining the traditional algorithm with BIM to realize second optimization and dynamic control of schedule. The concept of this new model involves (1) using OSM to express the overlapping relationships in a project schedule clearly, (2) getting precise overlap rates of coupled activities and crashing rates with an improved expression and utilization of chromosomes, (3) proposing an integrated framework of GA and BIM to prove the feasibility of the research in practice, and (4) providing the comparison of different optimization methods.

\section{Literature Review}

Overlapping is an effective tool widely used in different fields to reduce the completion time. There are two types of researches on overlapping: product development and project execution [7]. Since the1990s, a lot of researchers have investigated the inherent nature of overlapping and made great contributions to concurrency-based scheduling. Krishnan et al. [8] used two attributes like upstream evolution and downstream sensitivity to characterize activities and developed a detailed framework for coupled activities overlapping. The term evolution is defined as the refinement rate of upstream information from the preliminary to the final. The faster an evolution rate is, the earlier more accurate information can be released. The second characteristic sensitivity refers to how much a downstream activity is sensitive to possible changes in an upstream activity and how quickly it can accommodate those changes [7]. After studying the mechanism of overlapping for several years, Peña-Mora and Li [9] utilized the attributes and concepts in product development and modified them into parameters that were suitable for construction projects. There are also more researchers like Eppinger [10] and Blacud et al. [11] who have enquired into the concurrency attributes of construction activities and utilized the mechanism used in product development to develop an applicable framework to address activity overlapping in the construction industry. In Blacud's research, they focused on finding out which factors contributed more to the sensitivity of activities. To make the activity attributes in construction projects more clear, Bogus et al. [12] presented four determining factors (i.e., design optimization, constraint satisfaction, external information exchange, and standardization) that define a predecessor activity's evolution rate and three determining factors (i.e., constraint sensitive, input sensitive, and integration sensitive) that define the successor activity's sensitivity to the change of predecessor activities. And, Blacud et al. [11] advanced Bogus' research by interviewing multiple professionals in the construction industry and finally identified four determining factors (i.e., transformation processes, lead time, modularity, and interaction of components) to define the successor activity's sensitivity to the change of predecessor activities in a construction project. These researchers all dig deep into the attributes of overlapping activities. However, they did not investigate which activities should be overlapped to achieve the project goal effectively.

Project contract duration and project completion cost are two key objectives for the success of a project. So, there is a pressing need for the contractor to recognize these two objectives and achieve them effectively [13]. Therefore, many researchers have proposed various advanced methods to solve concurrency-based time-cost tradeoff problem. Roemer and Ahmadi [5] explained the rework generating from overlapping and evaluated the tradeoff between time and cost. Their research optimized overlaps of activities in a full chain other than only between two activities in the majority of researches. However, they did not investigate overlapping in a network of activities with multiple chains and predecessors. Several researchers have looked closely into activity overlapping in the construction industry and investigated the relationship between rework and the schedule overlapping. Some have advanced Krishnan et al.'s framework [8] and mechanism and adapted them to time-cost tradeoff problem in overlapped construction activities. Dehghan and Ruwanpura indicated that the rework probability increases as the time savings and the degree of overlapping increase [2]. Khoueiry et al. [14] and Srour et al. [15] developed a mathematical model that could compute the optimal overlapping strategy with the maximum profit after calculating the rework amount resulting from early start of successor activities. Dehghan and Ruwanpura $[2,6]$ have introduced a time-cost tradeoff model that determined the optimum overlaps considering resource constraints. Grèze et al. [3] applied overlapping into resource-constrained project scheduling, and Berthaut et al. [16] found a method to deal with time-cost tradeoff problem in resource-constrained project scheduling problems in the situation of overlapping. Besides, Hazini et at. $[17,18]$ hybridized activity overlapping with techniques like simulation for the solution of rework probability and presented a heuristic method to obtain the maximum profit of schedule compression with activity overlapping in conjunction with activity accelerating. Dehghan and Ruwanpura [2, 6] presented a model of tradeoff between activity overlapping and rework of design activities considering multipredecessors and cascades of overlaps. They conducted lots of studies focusing on the rework costs and benefits of activity overlapping and the method to formulate these factors. The most recent research was 
conducted by Gwak et al. [19]. This research provided a computational method that recognized the exact overlap duration between activities, considered the dynamic changes of critical activities, handled overlapping of activities with multiple predecessors and/or successors, and provided a mathematical formula that calculated the rework amount and cost. In addition, the duration compressed by overlapping is limited on account of the maximum overlap rates. So the time-cost tradeoff problem in crashing also deserves research. Most studies use linear programming to solve this problem. Since 1961, Fulkerson [20] proposed a linear method to figure out the lowest project cost. Then, many researchers have applied integer linear programming or dynamic programming in this problem [21, 22].

However, the existing studies related to time-cost tradeoff analysis in overlapping has not reached academic and practical maturity [12]. Indeed, these researchers all apply a fixed overlap rate (e.g., the overlap rate is set as $20 \%$ for all activities) to all coupled activities in their studies, which may result in much deviation. And, the linear programming method in the research of crashing does not conform to the practical conditions. Overlapping can shorten project duration with relatively low cost, but the compression degree is limited. Crashing can compress the schedule a lot, but the accompanying cost is high. Therefore, existing time-cost tradeoff analyses in schedule compression field lack academic and practical value because they do not offer mature exact overlap strategy and seldom consider the combination of crashing. This study presents a new method to deal with the problem of time-cost tradeoff scheduling. The model proposed in this paper illustrates the dependency relationship of activities with Overlapping Strategy Matrix (OSM). It analyzes various attributes and their impacts on overlapping and finally refines the overlap rate with optimized genetic algorithm. The new method described in this article complements the deficiencies of existing studies and combines overlapping with crashing to output an optimized schedule. Besides, the improved method of calculating optimal overlapping strategy in time-cost tradeoff problem is a theoretical research. It is difficult to prove its feasibility in implementation. To solve this problem, this paper proposes an integrated framework of GA and BIM. The framework uses the visualization function and integration module of BIM to analyze the feasibility of the optimized schedule in practical projects. Practitioners can realize dynamic schedule optimization and control after this framework being developed.

\section{Methods and Analysis}

3.1. Overlapping Principle. Figure 1 shows two overlapped activities with information dependency. The progress of a successor activity depends on the predecessor activity, for the successor can be started only if the information generated by the predecessor can be obtained (e.g., the successor activity can be pouring concrete by the workers and the predecessor activity can be preparing distribution drawings of columns). To compress the completion time, once the

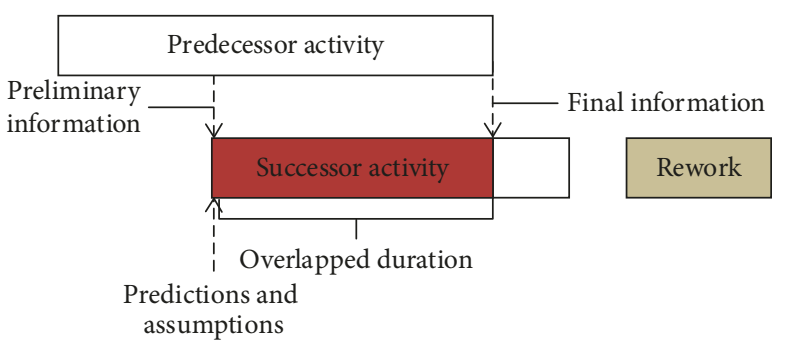

Figure 1: The mechanism of overlapping.

predecessor activity releases preliminary information, the successor activity is intentionally started right away with some preliminary predictions and assumptions. However, while the predecessor is completed, the final information released by it always differs from the preliminary or intermediate information, and therefore rework appears to make it compatible with the final information. It means extra person-hours (i.e., extra cost and time) of the successor activity compared to its normal duration [2]. A noteworthy point is that there exists a maximum allowable overlapping duration. Further overlapping is actually impossible for the lack of preliminary information. And, in this paper, it is defined by the maximum overlapping rate $\left(O_{\mathrm{M}}\right)$. The corresponding minimum overlapping rate $\left(O_{N}\right)$ is set as 0 , which means no preliminary information is released by the predecessor activity. Apart from some really exceptional cases, the degree of overlapping and the rework amount have a positive correlation. The more the two activities overlap, the less the confirmed information can be obtained by the successor activity, thus leading to larger rework probability and rework amount.

3.2. Overlapping Strategy Matrix. Overlapping Strategy Matrix (OSM) is first introduced by Hossain [23] to get an optimal overlapping strategy. OSM is derived from the Design Structure Matrix (DSM), an efficient technique in describing and the information flows between construction activities [24]. Figure 2 shows how OSM is derived from DSM. In traditional DSM, each "1" represents the dependency of row activity on the corresponding column activity. But, the traditional finish-start schedule always tends to far exceed the duration required by contract. To shorten the duration, the schedulers should start an activity once it has received preliminary parameters from the predecessor. It is assumed that all activities can get preliminary information from its predecessor activities for illustration purpose. And, all activity pairs able to overlap are shaded in the matrix, whether it is overlapped or not in the end. In this condition, " 1 " is changed to " 0 " in the OSM, which indicates that the information from predecessor activity is estimable and the successor activity can proceed with incomplete preliminary information from the predecessor activity and the final information will be checked upon its completion. Since OSM can show the potential of overlapping between activities, it is more suitable than DSM for calculating the exact overlap rates in the optimal result. 


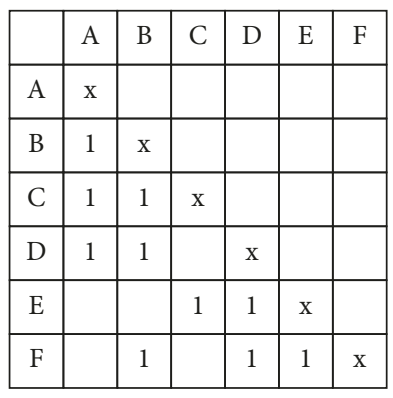

(a)

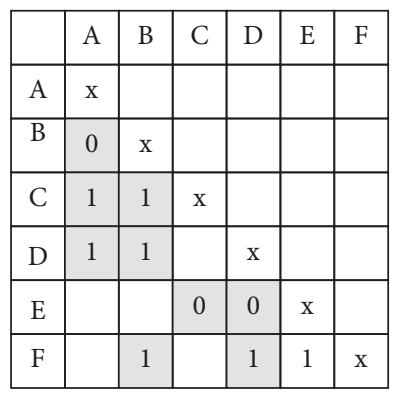

(b)
FIgure 2: The comparison of OSM and DSM.

3.3. Optimization Approach. Since both duration and cost have to be minimized, overlapping time-cost tradeoff is a multiobjective optimization problem. Some researchers $[6,18]$ have proposed applicable overlapping time-cost tradeoff objective functions to solve this problem. In this paper, the authors formulate an objective function referenced on previous studies as follows:

$$
\begin{aligned}
\operatorname{minimize} C= & \sum_{i=1}^{n} C_{\mathrm{D}}(i)+R \times C_{\mathrm{I}}(j) \\
& + \begin{cases}\mathrm{pc}\left(T-T_{\mathrm{cd}}\right), & \text { if } T \geq T_{\mathrm{cd}}, \\
0, & \text { if } T<T_{\mathrm{cd}},\end{cases}
\end{aligned}
$$

where $C$ is the total cost of the project, including duration compression and rework time caused by overlapping, $C_{\mathrm{D}}(i)$ is the direct cost of activity $i$ without considering overlapping, $R$ is the total rework duration caused by the changes of predecessor activities, $C_{\mathrm{I}}(j)$ is the daily indirect cost of successor activity $j$ originated from rework, including daily wage and other fees, $T$ is the project total duration, $T_{\text {cd }}$ is the contract duration of the project, and pc means the penalty coefficient when the completion date of a project is delayed, which is subject to $p c \geq 0$ and $T_{c d}>0$.

Equation (1) represents that the total cost of a project with an exact overlapping strategy consists of individual direct cost and extra rework cost, plus the penalty of late completion. Different from some other studies, a benefit coefficient of early completion is not adopted in this paper considering the practical condition in real-life construction. And, the penalty coefficient is set to eliminate inappropriate solutions exceeding the limited duration. Direct $\operatorname{cost} C_{\mathrm{D}}(i)$ in equation (1) is given in the case evaluated by experienced experts. Based on the mechanism of overlapping, both of rework probability $P_{i j}$ and rework duration $R_{i j}$ are functions of overlap length $L_{i j}$, as shown in equations (2), (6), and (7). According to the information and project experience suggested by Dehghan and Ruwanpura [2], the rework function is set as equation (3). According to the research from a large amount of practical construction data by Forcada et al. [25], rework cost is relevant to original contract value (OVC) and project location, as show in equation (4). OCV represents the project type, organization type, contract type, and original contract required duration comprehensively. Project location is of four types including local, regional, national, and international. To secure the reliability of the result, this paper uses the bigger one of equations (3) and (4) as final rework cost, as shown is equation (5). $P_{i j}$ is the rework probability which means the probability that a change of predecessor activity $i$ causes rework for successor activity $j$ and $0<P_{i j} \leq 1 . R_{i j}$ is the duration of rework added to successor activity $j$, resulting from the changes of predecessor activity $i$. And, as shown in equation (6), $L_{i j}$ is calculated by multiplying the overlap rate $\left(O_{i j}\right)$ and the successor activity's duration $\left(D_{j}\right)$ :

$$
\begin{aligned}
R_{i j}= & f\left(L_{i j}\right), \\
R_{i j}= & 0.2 L_{i j}, \\
\operatorname{Ln}\left(R^{\prime}\right)= & -2.502+0.628 \times \operatorname{Ln}(\mathrm{OCV}) \\
& +0.316 \times \text { project location, } \\
R= & \operatorname{Max}\left(\sum_{i=1}^{n} \sum_{j=1}^{m} P_{i j} R_{i j}, R^{\prime}\right), \\
L_{i j}= & O_{i j} \times D_{j} .
\end{aligned}
$$

The equivalent rework is defined by multiplying the rework probability $\left(P_{i j}\right)$ with the rework duration $\left(R_{i j}\right)$. In addition, pc should be big enough to get rid of strategies with long durations that exceed what is required by contract. Equation (7) is formulated based on the study of Gwak et al. [19] to evaluate the rework probability $P_{i j}$ to the successor:

$$
P_{i j}= \begin{cases}\left(\frac{O_{i j}}{3}\right)^{1 / 2}, & \text { if }\left(A_{\mathrm{E}}=S \& A_{\mathrm{S}}=L\right), \\ -1.6\left(O_{i j}\right)^{3}+2.4\left(O_{i j}\right)^{2}+0.01 O_{i j}, & \text { if }\left(A_{\mathrm{E}}=F \& A_{\mathrm{S}}=H\right), \\ \left(\frac{O_{i j}}{3}\right)^{1 / 3}, & \text { if }\left(A_{\mathrm{E}}=S \& A_{\mathrm{S}}=H\right), \\ -\left(O_{i j}\right)^{3}+1.5\left(O_{i j}\right)^{2}+0.01 O_{i j}, & \text { if }\left(A_{\mathrm{E}}=F \& A_{\mathrm{S}}=L\right) .\end{cases}
$$


In this function, $O_{i j}$ refers to the overlap rate between two activities. The $A_{\mathrm{E}}$ refers to the evolution rate of a predecessor activity and the $A_{\mathrm{S}}$ refers to the sensitivity and rework probability resulted from changes of a successor activity. $\mathrm{F}$ and $\mathrm{S}$ represent that the evolution rate of an activity is fast or slow. $\mathrm{H}$ and $\mathrm{L}$ represent that the sensitivity and rework probability of the successor activity is high or low. Rework probability is directly related to the overlapping rate, since the uncertainty increases with the degree of overlapping. Meanwhile, the correlation between $P_{i j}$ and $O_{i j}$ is not always the same so that the corresponding formula differs in the aspect of attributes like evolution and sensitivity. As shown in Figure 3, if the evolution rate of an activity is slow, less accurate information can be released and transferred to the downstream activities, leading to a higher risk and probability of rework. As for the influence of sensitivity, if the sensitivity of an activity is low, the rework probability will also be low since external change has less influence on an activity with low sensitivity. Given the overlapping rate, evolution rate, and sensitivity, the rework probability to the successor activity can be computed using the corresponding function from equation (7). This equation is simulated from Figure 3 and is obtained through professional judgment on a project-by-project basis. The correlation between overlapping and rework has been studied by many researchers (e.g., $[4,12])$. Figure 3 from the study of Han-Seong Gwak shows the correlation between overlap and rework under different circumstances of evolution and sensitivity. It reveals that there is a positive correlation between the probability of rework and the degree of overlapping.

Meanwhile, in the resource collaborative crashing condition, project duration and project coast after crashing can be represented as follows:

$$
\begin{aligned}
& D_{i s} \approx \frac{D_{i}}{\eta_{i} n_{i}}=n_{i}^{\alpha_{i}} \frac{D_{i}}{n_{i}}=n_{i}^{\left(\alpha_{i}-1\right)} D_{i} \longrightarrow n_{i} \approx\left(\frac{D_{i \mathrm{~s}}}{D_{i}}\right)^{1 /\left(\alpha_{i}-1\right)}, \quad \text { (8) } \\
& C_{i s}=C_{i \mathrm{r}}+n_{i}\left(m_{i}+D_{i \mathrm{~s}} v_{i}\right) \approx C_{i \mathrm{r}}+\left(\frac{D_{i \mathrm{~s}}}{D_{i}}\right)^{1 /\left(\alpha_{i}-1\right)}\left(m_{i}+D_{i \mathrm{~s}} v_{i}\right),
\end{aligned}
$$

where $D_{i s}$ represents the duration of activity $i$ after crashing. The performance coefficient $\eta_{i}$ represents the loss of resource efficiency caused by coworking multiresources in activity $i . n_{i}$ represents the number of resources involved in activity $i$. And, $\alpha_{i}$ is between 0 and 1 . When $\alpha_{i}$ equals 0 , all resources in an activity are fully collaborated without any efficiency loss. And, when $\alpha_{i}$ equals 1 , the working efficiency of resources gets lower with the increase of the resource number. $C_{i s}$ represents the total cost of crashing of activity $i, C_{i r}$ represents the cost of nonrenewable resources, $m_{i}$ represents the cost of unit resource, and $v_{i}$ represents the unit variable cost.

However, in terms of the practical conditions, crashing will have bad effects on project quality inevitably. So, this paper defines the project quality with $Q$ and it is represented as follows:

$$
\begin{aligned}
& Q=a T^{2}+b T+c, \\
& \left\{\begin{array}{l}
a T_{\mathrm{s}}^{2}+b T_{\mathrm{s}}+c=Q_{\min }=50 \%, \\
a T_{\mathrm{m}}^{2}+b T_{\mathrm{m}}+c=Q_{\min }=80 \%, \\
a T_{1}^{2}+b T_{1}+c=Q_{\min }=100 \%,
\end{array}\right. \\
& T_{1}=T_{s}+0.618\left(T_{1}-T_{s}\right), \\
& C_{\mathrm{s}}=\sum_{i=1}^{n} C_{i \mathrm{f}}+\frac{1}{\mathrm{Q}} \times \sum_{i=1}^{n} C_{i \mathrm{v}},
\end{aligned}
$$

where $T$ represents the total project duration and $a, b$, and $c$ are determined with equation (11). $T_{\mathrm{s}}$ represents the shortest duration, $T_{1}$ represents the longest duration, and $T_{\mathrm{m}}$ represents the optimal duration, as determined by equation (12). Under this assumption, the total cost is finally represented as equation (13).

The above paragraphs illustrate that there are a great amount of possible scheduling strategies. Therefore, the process of continuous optimization will be tedious and complicated. In the following section, a hybridized optimization algorithm is introduced and explained to figure out the multiparametric problem. The algorithm is designed to assess all kinds of overlapping strategies and makes the optimal decision.

3.4. Optimization Algorithm. There are two different scenarios in the time-cost tradeoff problem. The first one is when the project contract duration $\left(T_{c d}\right)$ is given to find an overlapping strategy that generates the minimum cost. The other is to find an overlapping strategy which leads to the minimum duration with a fixed project target cost [6]. The main objective of this paper is to figure out an improved method to solve the first situation. In this section, the optimization of the coding way in chromosome is explained.

In this combinatory research model, the researchers begin by developing a schedule optimization model to facilitate scheduling, then get all the information needed about some cases, and express the dependency relationship of activities using the DSM. After that, the OSM can be derived from a list of estimable activities and the DSM. In the OSM, each dependency relationship is expressed by tens of genes to figure out the exact overlap rate as well as crashing rate and then all of them form a chromosome. After achieving all information of the project, GA initializes ' $n$ ' solutions through stochastic universal sampling selection way for the set project and transmits them back to OSM. The schedules can be generated with certain values of the rework duration and rework cost for the objective functions. The GA algorithm then evaluates each solution based on certain values and conducts fitness evaluation. Afterwards, the parents are selected based on the fitness to generate offspring population through single-point crossover and mutation operations. The results are then taken over by the scheduling model to generate new schedules which are again evaluated. The 


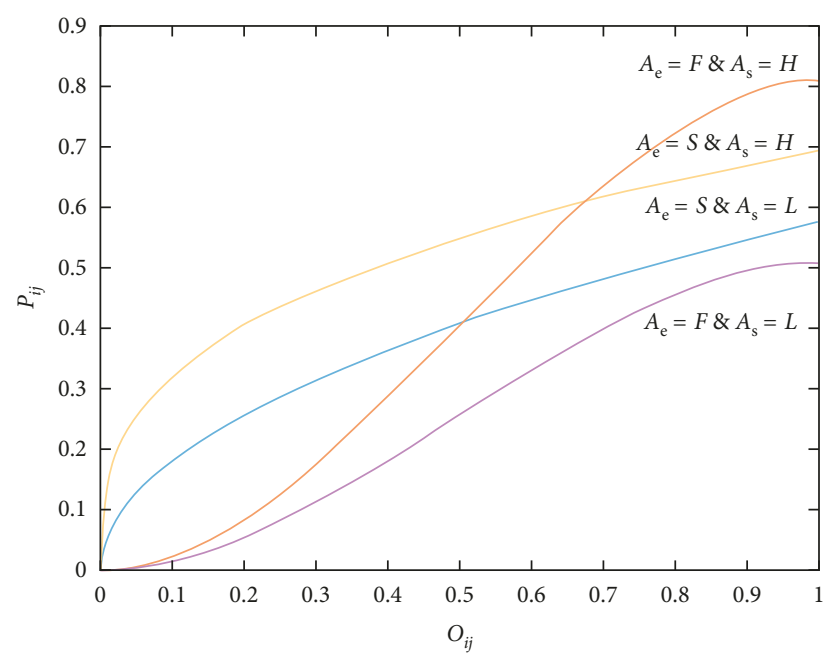

FIGURE 3: Correlation between overlapping rate and rework probability under different attributes.

whole process ends when one of the stopping criteria of GA is met up with and the optimization model tells both the best solution and all values in that schedule. In the case of this paper, the process ends when it has evolved for 1000 generations.

The algorithm generates a collection of random overlapping strategies, and each of them encompasses several overlap rates, the number of which is the same as dependency relationships. While in the binary coding system, the gene can only represent " 0 " or " 1 ", which means the result can only be "Not overlap" or "Overlap". Hence, in the existing researches, a fixed overlap rate is given to all overlap activities for calculation. To make the calculation more accurate, this research proposes a new method to express the chromosome creatively. Each gene in the previous method is further represented by tens of binary numbers. Therefore, the original result of "overlap or not overlap" can be refined to exact overlap rates as percentages. Likewise, the exact crashing rate can be obtained by the algorithm. For the convenience of illustration, Figure 4 shows a random chromosome using only 9 activities from the case in Table 1.

Apart from these variables in objective function, there are also some genetic algorithm parameters to be set. These variables can largely affect the efficiency of calculation. For the case in this paper, the number of individuals (NIND) means the number of project schedules in the scheduling context and is set as 100 to make sure the population size is big enough to decrease the error in random selection overlapping strategies. The maximum generation (MAXGEN) is 1000, which means the process of selecting, crossing, and mutating random overlapping strategies will iterate 1000 times for a relatively accurate and optimal solution. In the scheduling problem, a chromosome represents a solution of overlapping strategy and a gene means an exact overlap rate in the strategy. Hence, the length of chromosome (NVAR) in the scheduling context depends on the number of dependency relationships in a project, which in this case is 23 , and the optimal overlapping strategy contains 23 exact overlapping rates. And, the precision of variables (PRECI) represents the accuracy class in refining the overlap rates and is set as 20 in the case of this paper. The specific explanation of chromosome is further described in the next subsection. In addition, the gap (GGAP) is defined as 0.9 , while the crossover probability $\left(p_{\mathrm{x}}\right)$ and the mutation probability $\left(p_{\mathrm{m}}\right)$ are 0.7 and 0.01 , respectively.

3.5. The Application Combining GA with 4D BIM. Since the optimization process described above is based on theoretical research, this paper proposed a brand new thought of combing the GA and BIM platform to prove the practical meaning of the research. As can be seen in Figure 5, the framework of schedule optimization and dynamic control based on GA and BIM mainly contains four steps:

(1) Optimize the project schedule using the improved objective function and genetic algorithm, and then optimize the result for the second time by integrating with $4 \mathrm{D}$ BIM visualization.

(2) Implement the schedule and track the onsite data and project requirements, and then analyze whether the project need adjustment.

(3) If the schedule need adjustment due to delay or requirement change, optimize the schedule again with the updated information.

(4) If there are any changes in the building, modify the BIM model according to the newest design and then analyze the unreasonable schedule or conflicts through dynamic simulation. Finally output the optimal schedule and realize the dynamic loop control of schedule.

The advantages of integrating GA with BIM come from four aspects:

(1) The visualization function of BIM can represent the schedule intuitively, showing the probable conflicts in project schedule. The project participants can then estimate the feasibility of the schedule. 


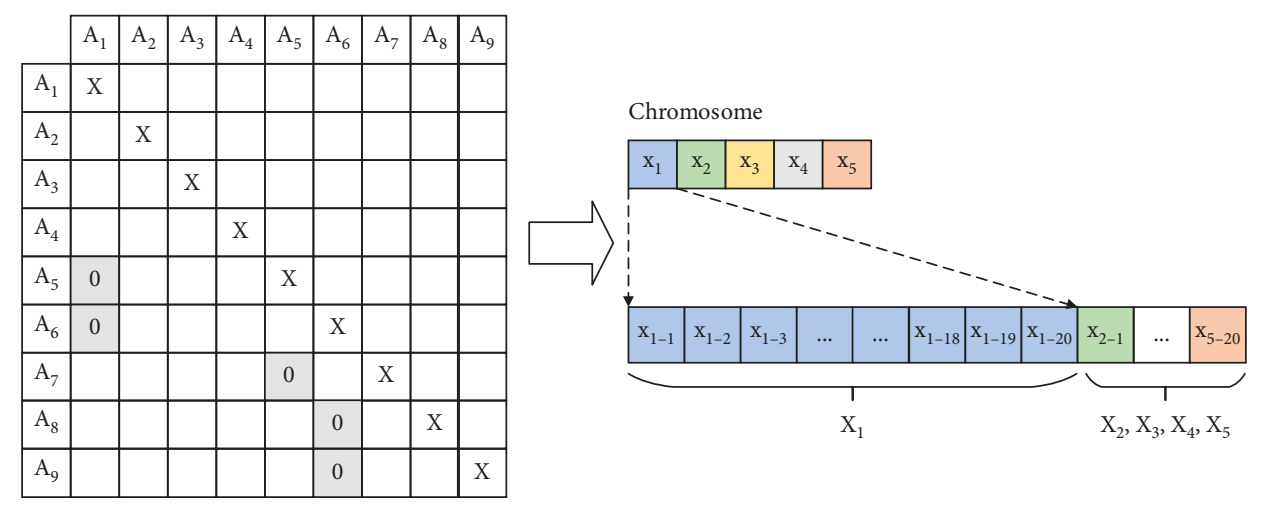

FIGURE 4: The genes in a random chromosome.

TABLE 1: Information of activities' attributes.

\begin{tabular}{|c|c|c|c|c|c|c|c|c|c|c|}
\hline Activity & Predecessors & $D_{i}$ (days) & Direct cost (dollars) & $A_{\mathrm{E}}$ (evolution) & $A_{\mathrm{S}}$ (sensitivity) & $O_{\mathrm{N}}$ & $O_{M}$ & $\alpha_{i}$ & $m_{i}$ & $v_{i}$ \\
\hline 1 & - & 24 & 2,400 & Fast & High & - & - & 0.5 & 25 & 12 \\
\hline 2 & - & 25 & 1,000 & Slow & & - & - & 0.333 & 12 & 25 \\
\hline 3 & - & 33 & 3,200 & Slow & Low & - & - & 0.5 & 12 & 25 \\
\hline 4 & - & 20 & 30,000 & Fast & Low & - & - & 0.333 & 25 & 12 \\
\hline 5 & 1 & 30 & 15,000 & Fast & High & 0.0 & 0.65 & 0.5 & 12 & 38 \\
\hline 6 & 1 & 24 & 40,000 & Slow & High & 0.0 & 0.3 & 0.333 & 50 & 25 \\
\hline 7 & 5 & 18 & 22,000 & Fast & High & 0.0 & 0.5 & 0.666 & 25 & 12 \\
\hline 8 & 6 & 24 & 120 & Slow & Low & 0.0 & 0.5 & 0.5 & 12 & 12 \\
\hline 9 & 6 & 25 & 300 & Slow & Low & 0.0 & 0.6 & 0.333 & 25 & 25 \\
\hline 10 & 2,6 & 33 & 450 & Slow & Low & 0.0 & 0.8 & 0.666 & 25 & 12 \\
\hline 11 & 7,8 & 20 & 350 & Slow & High & 0.0 & 0.6 & 0.5 & 12 & 25 \\
\hline 12 & $5,9,10$ & 30 & 2,000 & Fast & Low & 0.0 & 1 & 0.5 & 12 & 38 \\
\hline 13 & 3 & 24 & 1,800 & Slow & High & 0.0 & 0.5 & 0.666 & 25 & 12 \\
\hline 14 & 4,10 & 18 & 2,200 & Slow & Low & 0.0 & 0.75 & 0.5 & 12 & 12 \\
\hline 15 & 12 & 16 & 4,500 & Slow & High & 0.0 & 0.8 & 0.333 & 25 & 25 \\
\hline 16 & 13,14 & 30 & 1,000 & Fast & High & 0.0 & 0.65 & 0.5 & 12 & 38 \\
\hline 17 & $11,14,15$ & 24 & 4,000 & Slow & Low & 0.0 & 0.7 & 0.666 & 50 & 12 \\
\hline 18 & 16,17 & 18 & 3,000 & Fast & High & 0.0 & 1 & 0.5 & 25 & 25 \\
\hline
\end{tabular}

(2) After estimation, the scheduler can make some adjustments and optimize the schedule for the second time.

(3) Engineers can collect onsite data, track the project requirements, and upload all information on the real-time integrated $4 \mathrm{D}$ BIM platform, so that the BIM platform can analyze the schedule variance and adjust the schedule dynamically according to practical conditions.

(4) BIM platform can store a large amount of data relevant to schedule management effectively, making it possible to generate and recommend schedule automatically through data mining in the future.

\section{Case Results and Discussion}

4.1. Case Results. To investigate the practicability and applicability of the method in the real-world construction project, an illustrative sample is presented herein to explain the proposed algorithm with full details and appreciate the impact on total project duration and total amount of project cost. This case was originally reproduced from $[13,26]$ and was used in an existing study [19] to introduce the results of their research. The intention of using the same case in this paper is to make it convenient for the interested readers to further understand and compare these algorithms or apply them to similar problems. After the illustration, a comparison of results achieved by different methods is provided.

Table 1 depicts a list of 18 activities with necessary information. The amount of rework generated by each pair of overlap and the estimated cost of each pair of overlap can be obtained through the functions provided in the preceding section including equations (1), (4), and (5). And, the overlap rate $\left(O_{i j}\right)$ represents the percentage of activity $j$ that is in parallel with activity $i$. In Table 1 , the overlap rate has an upper bound value defined as the maximum overlap rate $\left(O_{\mathrm{M}}\right)$ and a lower bound value defined as the minimum overlap rate $\left(O_{N}\right)$. It has been assumed that the information from any predecessor in this case is estimable, and the estimated early information can be transmitted from the predecessor to the successor. To some extent, the maximum overlap rate represents the time when early information can be obtained. For instance, if the maximum overlap rate of an activity is 0.65 , it means that the estimated information cannot be transmitted until 35\% of the predecessor activity 


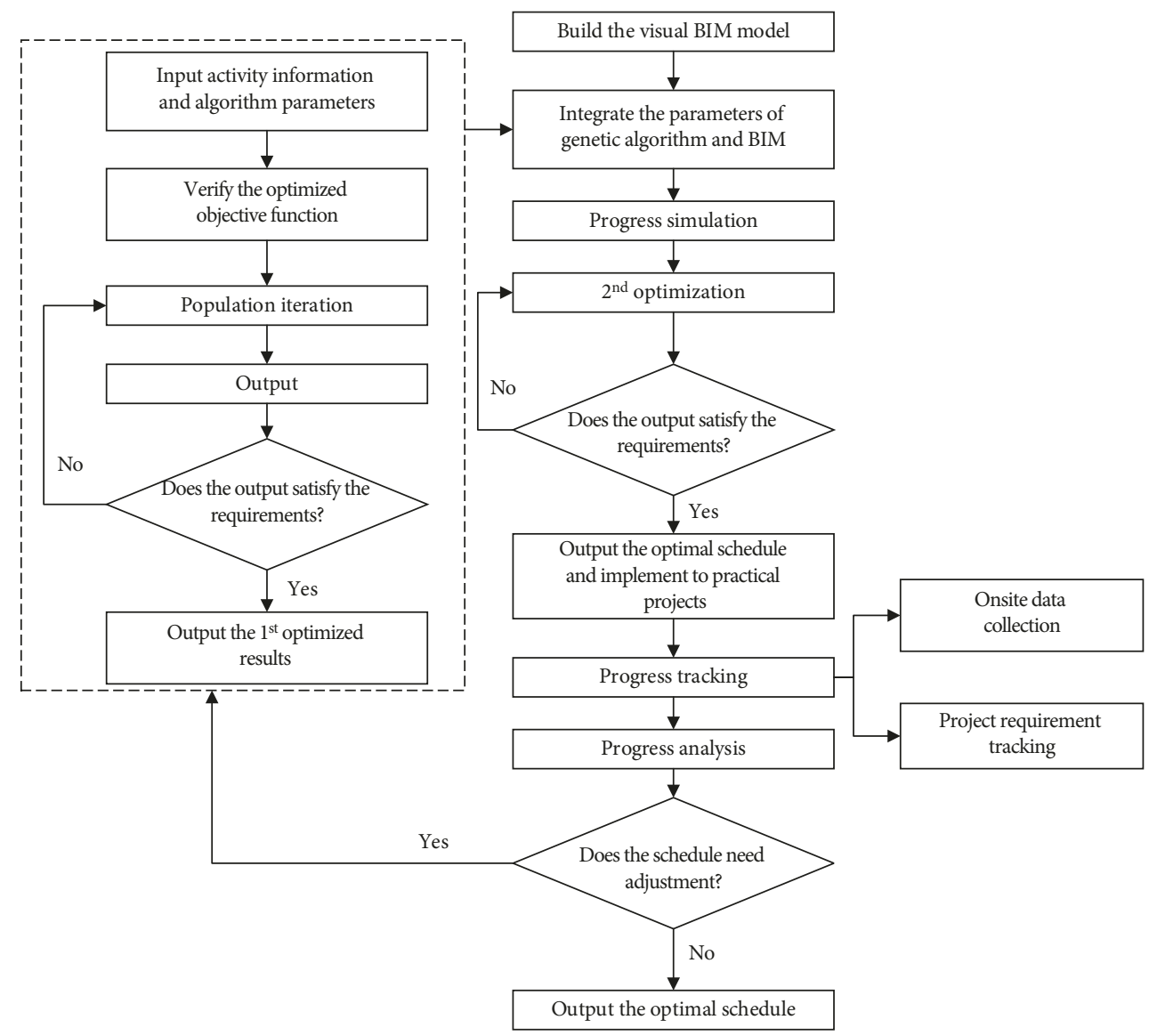

Figure 5: Dynamic schedule optimization and control based on GA and BIM.

has been completed. In this case, the daily indirect cost $\left(C_{\mathrm{I}}(j)\right)$ is set as $\$ 63 /$ day and the project duration required by contract $\left(T_{c d}\right)$ is 110 days. Without optimization, the total project completion time on the critical path of $(1 \longrightarrow 6 \longrightarrow 10 \longrightarrow 12 \longrightarrow 15 \longrightarrow 17 \longrightarrow 18)$ of normal schedule with no overlap is 169 days. Accordingly, the total project completion cost is $\$ 143,967$, calculated from total direct cost $(\$ 133,320)$ plus indirect cost $(\$ 63 /$ day $\times 169$ days).

The dependency relationship of this project can be represented intuitively using the OSM matrix in Figure 6. Since all the information from predecessor activities are estimable in this case, all the elements used in the shaded cells are "0", which means the corresponding activities are able to start earlier with estimated information.

For the case example, the population with 23 solutions has evolved for 1000 generations to produce an approximately optimal schedule. The crossover probability is set to 0.7 and the mutation probability in GA optimization is set to 0.01 . In addition, the targeted completion date of the project is 110 days and the penalty coefficient is set to 2 so that expected project completion duration remains within project contract date. Setting of penalty coefficient will discard those schedules with completion time higher than the project contract date.

To understand how optimal overlapping strategy can optimize the project schedule better, Figure 7 shows

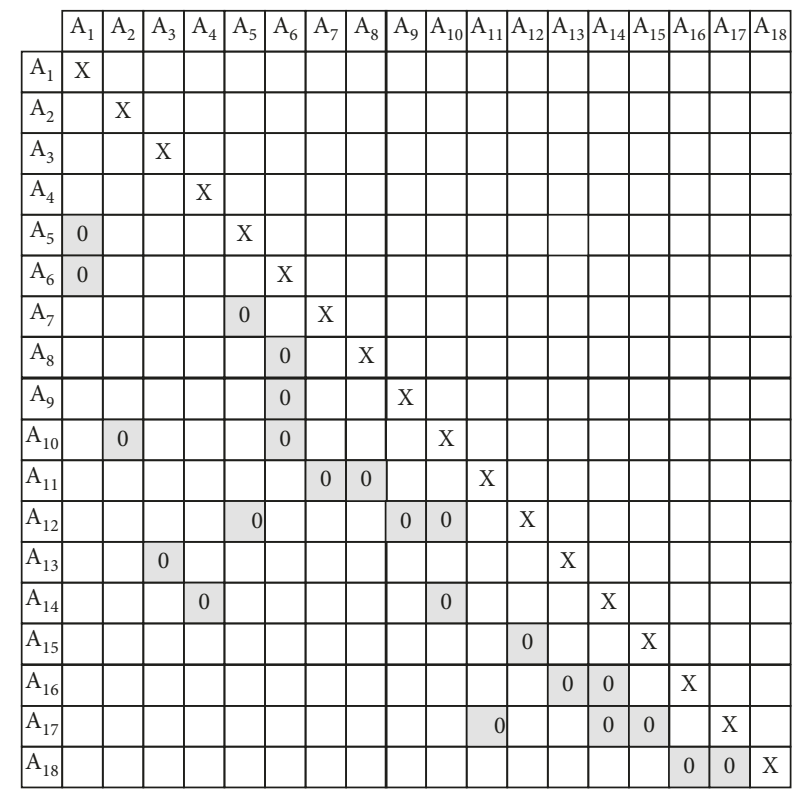

Figure 6: The OSM matrix of the case.

scheduling results of four overlapping scenarios. In this figure, TD represents total duration and TC represents total cost. The first scenario represents the original schedule without optimization and overlapping. All activities do not 


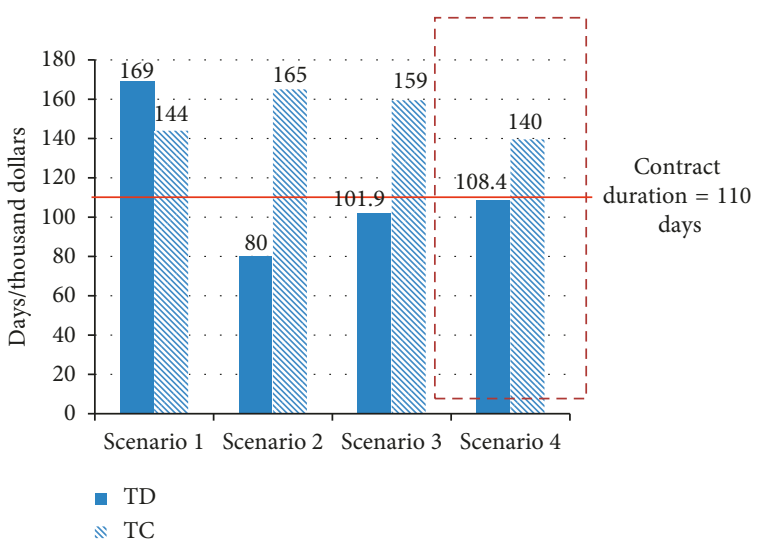

Figure 7: Comparison of four overlapping scenarios.

start until the predecessor activities are completed. The total duration is 169 days, and the total cost is $\$ 143,967$. In the second scenario, each activity starts once it receives early information released from all of its predecessors. In this scenario, project completion time can be significantly compressed to 80 days with the total cost of $\$ 165,006$. There is a remarkable reduction (39.7\%) of duration compared to scenario 1 . However, the total cost increases quite a lot that makes it inappropriate for practical projects. The third scenario represents the result computed in the original study of this case, in which the project completion time is 101.9 days $(39.7 \%$ reduction) and the project completion cost is $\$ 159,425$, respectively. The last scenario shows the result of the optimal overlapping strategy where only activities which can shorten total completion duration with minimal rework amount are overlapped with appropriate and exact overlap rates. The strategy obtained with the optimization method significantly eliminates the amount of total duration and cost $35.9 \%$ and $3.1 \%$ reduction compared to scenario 1 , respectively) without delaying overall duration. In this scenario, the total duration is 108.4 days and the total cost is $\$ 139,539$, as is shown in Figure 7 . Table 2 shows the exact overlapping strategy of the illustrative case. The overlap rates are output by the algorithm, and other results are calculated by the formulas in the method.

The aforementioned discussion shows the difficulty of determining an optimal overlapping strategy. The superiority of the optimized method is mainly represented in two aspects: (1) the total duration is much shorter than the one before optimization. Although it is a bit longer than the results in scenario 2 and scenario 4 , the duration satisfies the contract required date and makes the overlapping strategy acceptable. (2) the total duration is largely reduced compared to other scenarios since the proposed model can eliminate unnecessary rework. This kind of optimization is of value to practical construction projects.

Under the circumstances of applying the combinatory method, this paper assumes that overlapping and crashing is independent and does not consider the mutual influence. Table 3 shows the optimization result of the combinatory method. Table 3 shows the duration after crashing and resources requirement of each activity. Table 4 shows the exact overlap rates of each activity pair. Compared to Table 2, overlap rates are apparently lowered, inducing less rework and effect on total cost. The duration after optimization is 52.7 days with the total cost of 150,562 dollars. It is obvious that applying overlapping and crashing comprehensively can shorten the duration to a large extent if necessary, and the total cost will be lower than using crashing method only.

4.2. Discussion. The preceding sections clearly demonstrate a systematic schedule optimization strategy that can obtain a tradeoff between reduction in project completion duration and increase of cost. As the obtained result reveals, the rework probability and rework duration increase as the sensitivity to change in upstream activities increases, which is consistent with the hypothesis of Bogus et al. [12] and Blacud et al. [11]. The evolution rate of upstream activities and the sensitivity of downstream activities are incorporated in the case to identify the accuracy of early estimated information and the rework probability. The construction activities with slow evolution rate and high sensitivity should be assigned with less overlap rates to eliminate unnecessary rework.

Finally, the proposed model based on hybridized algorithms provides an optimized approach to formulate a detailed construction schedule within the required contract duration. The project manager is given the optimization model, duration and dependency information, activity attributes, and the overlap constraints to make the schedule for the construction project case. And, when the contractor has an extremely strict requirement on project duration, the scheduler can apply overlapping and crashing comprehensively to utilize the advantage of each method to the largest extent. Then, the result of first optimization can be integrated with $4 \mathrm{D}$ BIM platform to simulate the schedule with visualization function. This kind of integration makes it more intuitional and dynamic to observe the implementation of optimized schedule. The practitioners can analyze the correlation between components and activities, especially those complicated processes, to make the theoretical schedule coincide more with the practical conditions in projects. The second optimization step ensures the rationality and practicality of the optimal schedule. Taking the construction joint and settlement joint as an example, according to the experiences, the influence and propagation of cross construction between these two processes are great due to the complexity of operation and large number of embedded parts. Therefore, after observing this condition in the BIM model, the practitioners can adjust the overlap rate between these two activities in the input parameters and obtain an updated schedule with exact overlap rates. After second optimization, the schedule can be implemented and onsite data can be tracked and updated in real time, finally realizing dynamic control of schedule.

Through all these phases and activities, this research finally creates a virtuous cycle in which researchers can achieve a satisfactory result and improve the optimization tool at the same time. 
TABLE 2: The optimal overlapping strategy of the case.

\begin{tabular}{|c|c|c|c|c|c|}
\hline Overlap activity & Overlap rate & Overlap duration (days) & Rework duration (days) & Rework probability & Rework cost (dollars) \\
\hline $1-5$ & 0.218026 & 6.54 & 1.31 & 0.10 & 256.69 \\
\hline $1-6$ & 0.299989 & 7.20 & 1.44 & 0.46 & 388.77 \\
\hline $5-7$ & 0.21767 & 3.92 & 0.78 & 0.10 & 153.51 \\
\hline $6-8$ & 0.499969 & 12.00 & 2.40 & 0.41 & 1079.86 \\
\hline $6-9$ & 0.599973 & 15.00 & 3.00 & 0.45 & 1619.86 \\
\hline $2-10$ & 0.799835 & 26.39 & 5.28 & 0.52 & 3800.03 \\
\hline $6-10$ & 0.799959 & 26.40 & 5.28 & 0.52 & 3801.21 \\
\hline $7-11$ & 0.599985 & 12.00 & 2.40 & 0.58 & 1295.93 \\
\hline $8-11$ & 0.599974 & 12.00 & 2.40 & 0.58 & 1295.89 \\
\hline $5-12$ & 0.285024 & 8.55 & 1.71 & 0.10 & 438.69 \\
\hline $9-12$ & 0.286209 & 8.59 & 1.72 & 0.10 & 442.35 \\
\hline $10-12$ & 0.285853 & 8.58 & 1.72 & 0.10 & 441.24 \\
\hline $3-13$ & 0.499996 & 12.00 & 2.40 & 0.55 & 1079.98 \\
\hline $4-14$ & 0.749908 & 13.50 & 2.70 & 0.50 & 1822.06 \\
\hline $9-14$ & 0.749951 & 13.50 & 2.70 & 0.50 & 1822.26 \\
\hline $12-15$ & 0.8 & 12.8 & 2.56 & 0.64 & 1843.2 \\
\hline $13-16$ & 0.217192 & 6.52 & 1.30 & 0.10 & 254.73 \\
\hline $14-16$ & 0.218397 & 6.55 & 1.31 & 0.10 & 257.57 \\
\hline $11-17$ & 0.699977 & 16.80 & 3.36 & 0.48 & 2116.66 \\
\hline $14-17$ & 0.699992 & 16.80 & 3.36 & 0.48 & 2116.75 \\
\hline $15-17$ & 0.699999 & 16.80 & 3.36 & 0.48 & 2116.80 \\
\hline $16-18$ & 0.216634 & 3.90 & 0.80 & 0.10 & 152.05 \\
\hline $17-18$ & 0.218784 & 3.94 & 0.79 & 0.10 & 155.09 \\
\hline
\end{tabular}

TABLE 3: The optimized duration and resource of each activity with the combinatory method.

\begin{tabular}{lcccccccccccccccccc}
\hline & 1 & 2 & 3 & 4 & 5 & 6 & 7 & 8 & 9 & 10 & 11 & 12 & 13 & 14 & 15 & 16 & 17 & 18 \\
\hline$D_{i s}$ (days) & 12.3 & 25.0 & 33.0 & 20.0 & 30.0 & 11.0 & 18.0 & 24.0 & 25.0 & 25 & 20 & 23.4 & 24 & 18 & 7 & 30 & 18.9 & 15.8 \\
$n_{i}$ & 3.8 & 1 & 1 & 1 & 1 & 3.2 & 1 & 1 & 1 & 2.4 & 1 & 1.6 & 1 & 1 & 3.5 & 1 & 2 & 2.3 \\
\hline
\end{tabular}

TABLE 4: The optimized overlap rate of each activity pair with the combinatory method.

\begin{tabular}{cccccccccccccccccccccccc}
\hline & \multirow{2}{*}{5} & $1-6$ & $5-7$ & $6-$ & $6-$ & $2-$ & $6-$ & $7-$ & $8-$ & $5-$ & $9-$ & $10-$ & $3-$ & $4-$ & $9-$ & $12-$ & $13-$ & $14-$ & $11-$ & $14-$ & $15-$ & $16-$ & $17-$ \\
& & & & 8 & 9 & 10 & 10 & 11 & 11 & 12 & 12 & 12 & 13 & 14 & 14 & 15 & 16 & 16 & 17 & 17 & 17 & 18 & 18 \\
\hline$O_{i j}$ & 0.01 & 0.30 & 0.02 & 0 & 0 & 0.03 & 0.80 & 0 & 0.01 & 0.01 & 0.03 & 0.30 & 0 & 0.01 & 0.01 & 0.80 & 0 & 0.01 & 0.01 & 0.02 & 0.70 & 0.03 & 0.21 \\
\hline
\end{tabular}

\section{Conclusion}

This paper presents a synthesized method that determines the optimal schedule with a set of exact overlap rates between coupled activities in the schedule network and exact crashing level. It considers the effect of evolution rate and sensitivity of activities on the rework probability and analyses the time-cost tradeoff using a hybridized algorithm. A mathematical formula capable of outputting the optimal overlapping strategy with total duration, cost, and exact overlap rates enumerative is further presented. Then, the combination with crashing and the corresponding time and cost functions are given. Given this optimization model, the project manager can make a better construction schedule precisely without exceeding the required contract duration. Besides, in the process of schedule compression, the project manager can also compare the cost and duration of different plans under various project conditions like schedule information, activity attributes, and contract requirements. For the contribution to exact calculation in compressed scheduling under project constraints involved in budget and time, this research advances the knowledge system relative to exact activity overlapping and crashing in the field of construction scheduling, thus also contributing a little to the construction industry. And, the integration with 4D BIM provides a new idea and research direction for future work.

However, there are still some limitations of the study presented in this paper which need further improvements. The limitations are as follows:

(1) Rework probability is calculated using an empirical formula in this research, while this calculation method may lead to inaccuracy of the result. Hence, in the future study, it would be commendable to simulate the rework probability in compliance with probability distributions like Poisson distribution.

(2) It may be an additional progress in exact scheduling to build time model and cost model separately. In order to balance the duration and cost, the time model can be built from the angle of overlapping and activities dependencies based on DSM and the cost 
model can be built considering the negation cost. The sophisticated models may produce activities overlapping plans of better elaboration and accuracy.

(3) It would be commendable to incorporate a change propagation predicting method to manage design changes in the multidisciplinary collaborative environment of construction. The exterior changes may lead to risk of completion within the contract duration. So, it may be encouraged to eliminate this kind of uncertainty using scientific modeling and analysis method.

\section{Notations}

$A_{\mathrm{E}}$ : $\quad$ Evolution of the activity

$A_{\mathrm{S}}$ : $\quad$ Sensitivity of the activity

C: $\quad$ Total cost of the project

$C_{\mathrm{D}}(i)$ : Total direct cost of activity $i$ without considering overlapping

$C_{\mathrm{I}}(j)$ : Daily indirect cost of activity $j$ resulting from rework, including daily wage and other fees

$L_{i j}$ : $\quad$ Overlapping duration between activity $i$ and activity $j$

$\mathrm{O}_{\mathrm{M}}$ : The maximum overlapping rate of the activity pair

$\mathrm{O}_{\mathrm{N}}$ : The minimum overlapping rate of the activity pair

$O_{i j}$ : Overlap rate of the activity pair

$P_{i j}$ : $\quad$ Rework probability of activity $j$ when a change occurs in activity $i$

pc: $\quad$ Penalty coefficient of delay

$R_{i j}$ : Rework duration of activity $j$ resulting from the change of activity $i$

$T: \quad$ Total duration of the project

$T_{\text {cd }}$ : Contract required duration of the project.

\section{Data Availability}

The paper is finished based on modeling. There are no extra data except for a case study.

\section{Conflicts of Interest}

The authors declare that they have no conflicts of interest.

\section{Acknowledgments}

This work was supported by a National Natural Science Foundation of China grant funded by the China government (No. 71671128). The contribution in project management, strategy, and information technology is gratefully acknowledged.

\section{References}

[1] J. E. V. Gerk and R. Y. Qassim, "Project acceleration via activity crashing, overlapping, and substitution," IEEE Transactions on Engineering Management, vol. 55, no. 4, pp. 590-601, 2008.

[2] R. Dehghan and J. Y. Ruwanpura, "The mechanism of design activity overlapping in construction projects and the time-cost tradeoff function," Procedia Engineering, vol. 14, pp. 19591965, 2011.

[3] L. Grèze, R. Pellerin, P. Leclaire, and N. Perrier, "CIGI2011: a heuristic method for resource-constrained project scheduling with activity overlapping," Journal of Intelligent Manufacturing, vol. 25, no. 4, pp. 797-811, 2012.

[4] T. A. Roemer, R. H. Wang, and R. Ahmadi, "Time-cost tradeoffs in overlapped product development," Operations Research, vol. 48, no. 6, pp. 858-865, 2000.

[5] T. A. Roemer and R. Ahmadi, "Concurrent crashing and overlapping in product development," Operations Research, vol. 52, no. 4, pp. 606-622, 2004.

[6] R. Dehghan and J. Y. Ruwnapura, "Model of trade-off between overlapping and rework of design activities," Journal of Construction Engineering \& Management, vol. 140, no. 2, article 04013043, 2014.

[7] R. Dehghan, K. Hazini, and J. Ruwanpura, "Optimization of overlapping activities in the design phase of construction projects," Automation in Construction, vol. 59, pp. 81-95, 2015.

[8] V. Krishnan, S. D. Eppinger, and D. E. Whitney, "A modelbased framework to overlap product development activities," Management Science, vol. 43, no. 4, pp. 437-451, 1997.

[9] F. Peña-Mora and M. Li, "Dynamic planning and control methodology for design/build fast-track construction projects," Journal of Construction Engineering and Management, vol. 127, no. 1, pp. 1-17, 2001.

[10] S. D. Eppinger, Three Concurrent Engineering Problems in Product Development, Seminar, Massachusetts Institute of Technology, Sloan School of Management, Cambridge, MA, USA, 1997.

[11] N. A. Blacud, S. M. Bogus, J. E. Diekmann, and K. R. Molenaar, "Sensitivity of construction activities under design uncertainty," Journal of Construction Engineering and Management, vol. 135, no. 3, pp. 199-206, 2009.

[12] S. M. Bogus, K. R. Molenaar, and J. E. Diekmann, "Concurrent engineering approach to reducing design delivery time," Journal of Construction Engineering and Management, vol. 131, no. 11, pp. 1179-1185, 2005.

[13] T.-K. Lim, C.-Y. Yi, D.-E. Lee, and D. Arditi, "Concurrent construction scheduling simulation algorithm," ComputerAided Civil and Infrastructure Engineering, vol. 29, no. 6, pp. 449-463, 2014.

[14] Y. Khoueiry, I. Srour, and A. Yassine, "An optimization-based model for maximizing the benefits of fast-track construction activities," Journal of the Operational Research Society, vol. 64, no. 8, pp. 1137-1146, 2013.

[15] I. M. Srour, M.-A. U. Abdul-Malak, A. A. Yassine, and M. Ramadan, "A methodology for scheduling overlapped design activities based on dependency information," Automation in Construction, vol. 29, no. 2, pp. 1-11, 2013.

[16] F. Berthaut, R. Pellerin, N. Perrier, and A. Hajji, "Time-cost trade-offs in resource-constraint project scheduling problems with overlapping modes," International Journal of Project Organisation and Management, vol. 6, no. 3, pp. 215-236, 2014.

[17] K. Hazini, R. Dehghan, and J. Ruwanpura, "A heuristic method to determine optimum degree of activity accelerating and overlapping in schedule compression," Canadian Journal of Civil Engineering, vol. 40, no. 4, pp. 382-391, 2013.

[18] K. Hazini, R. Dehghan, and J. Ruwanpura, "An evolutionary optimization method to determine optimum degree of activity accelerating and overlapping in schedule compression," 
Canadian Journal of Civil Engineering, vol. 41, no. 4, pp. 333-342, 2014.

[19] H. S. Gwak, S. H. Son, Y. J. Park, and D.-E. Lee, “"Exact time-cost tradeoff analysis in concurrency-based scheduling," Journal of Construction Engineering \& Management, vol. 142, no. 10, article 04016054, 2016.

[20] D. R. Fulkerson, "A network flow computation for project cost curves," Management Science, vol. 7, no. 2, pp. 167-178, 1961.

[21] W. L. Meyer and L. R. Shaffer, "Extending CPM for multiform project time-cost curves," Journal of the Construction Division, no. 91, pp. 45-68, 1965.

[22] C. Kang and B.-C. Choi, "An adaptive crashing policy for stochastic time-cost tradeoff problems," Computers \& Operations Research, vol. 63, pp. 1-6, 2015.

[23] M. A. Hossain, Studying the physics of design flow incorporating early information using a simulation model [Ph.D. Thesis], National University of Singapore, Singapore, 2010.

[24] M. A. Hossain and D. K. H. Chua, "Overlapping design and construction activities and an optimization approach to minimize rework," International Journal of Project Management, vol. 32, no. 6, pp. 983-994, 2014.

[25] N. Forcada, M. Gangolells, M. Casals, and M. Macarulla, "Factors affecting rework costs in construction," Journal of Construction Engineering and Management, vol. 143, no. 8, article 04017032, 2017.

[26] T. Hegazy, "Optimization of construction time-cost trade-off analysis using genetic algorithms," Canadian Journal of Civil Engineering, vol. 26, no. 6, pp. 685-697, 1999. 


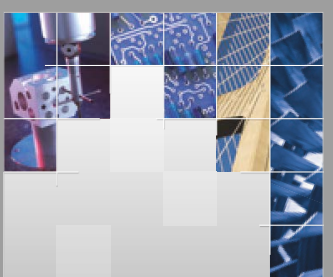

\section{Enfincering}
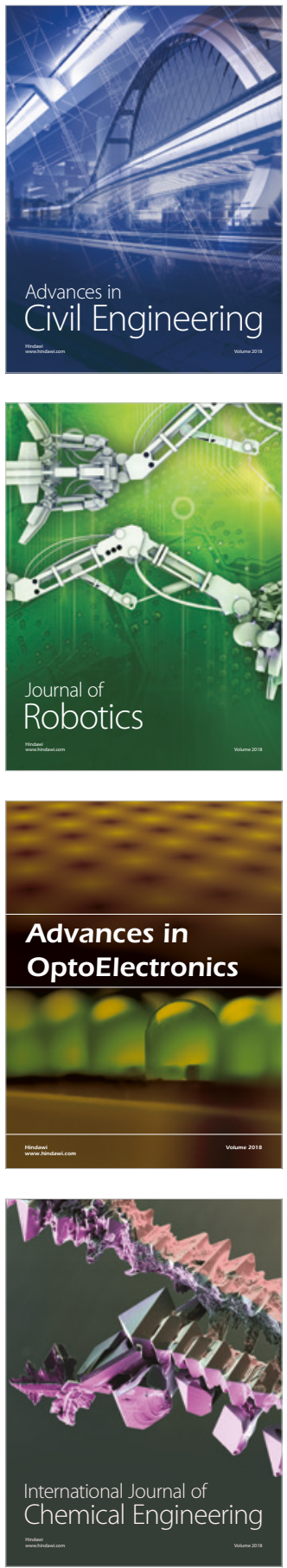

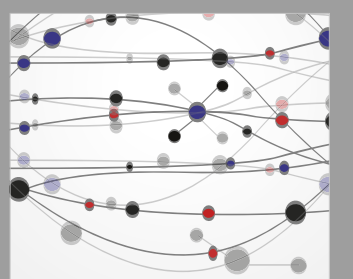

\section{Rotating \\ Machinery}

The Scientific World Journal

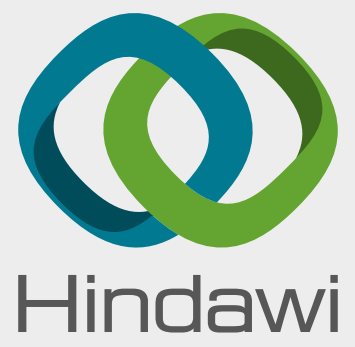

Submit your manuscripts at

www.hindawi.com
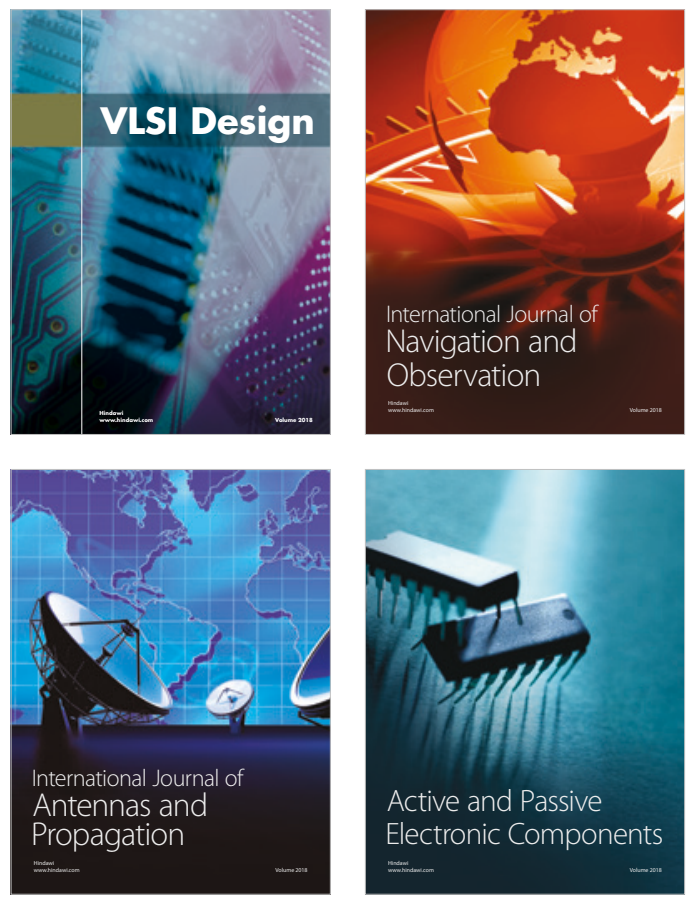
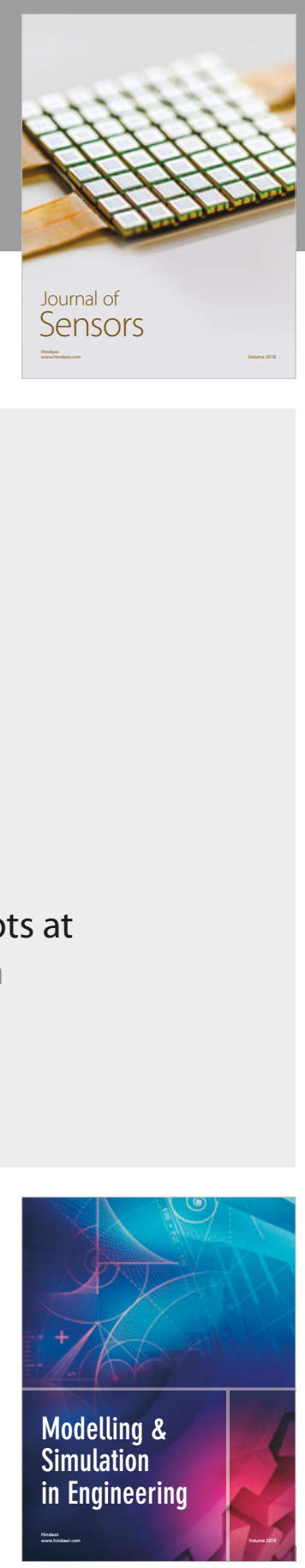

\section{Advances \\ Multimedia}
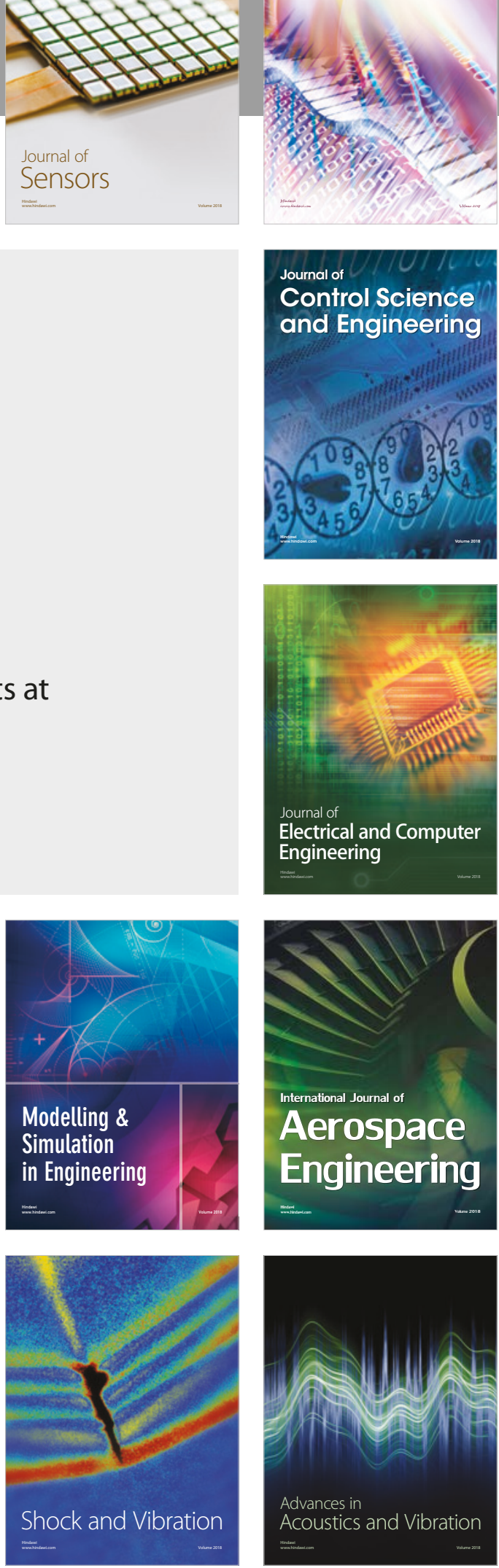